\section{Asella, Leben am Rande des großen Grabenbruchs}

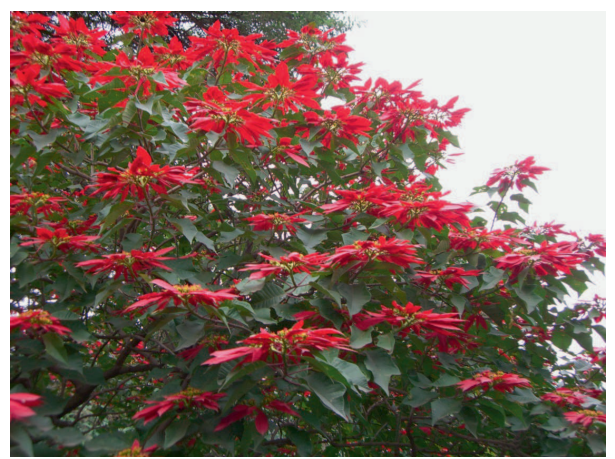

Beeindruckende Flora Äthiopiens. Die Weihnachtssterne (Euphorbia pulcherrima) wachsen als stattliche Bäume

\section{Die Stadt Asella}

Der Große Grabenbruch (engl. Rift Valley) erstreckt sich vom Norden Syriens über 6.000 km bis Mosambik. Die Breite des Tals variiert von 30 bis 100 km. Durch das Driften kontinentaler Platten entstanden über die letzten 35 Millionen Jahre tiefe Schluchten, wie das rote Meer, und hohe Gebirge, wie das äthiopische Hochland. Entlang des Grabenbruchs liegen noch viele aktive Vulkane, darunter der berühmte Erta Ale in Äthiopien, einer der weltweit sechs Vulkane mit einem immer aktiven Lava-See. Das äthiopische Hochland wird durch das Rift Valley in einen nordwestlichen und einen südöstlichen Teil gespalten. Von einigen Stellen an den Hängen des großen Grabens kann man einen atemberaubenden Blick über die Abbruchkante des Grabenbruchs in die Ferne genießen. Einer dieser Orte ist Asella. Die Stadt liegt auf $2.400 \mathrm{~m}$ über NN an den Hängen des 4.036 m hohen Mount Chilalo, der

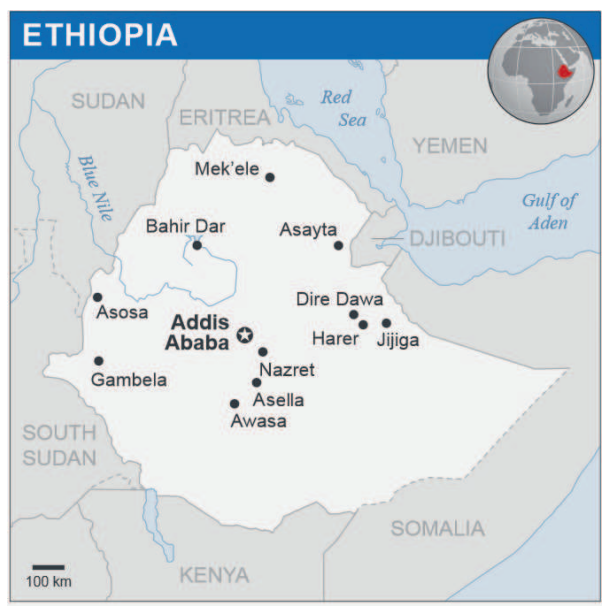

Äthiopien. Asella liegt südöstlich von Addis Abeba (Quelle: OCHA/ ReliefWeb). Nazret ist die Hauptstadt der Verwaltungsregion Oromia und wird in der Sprache der Oromo auch Adama genannt

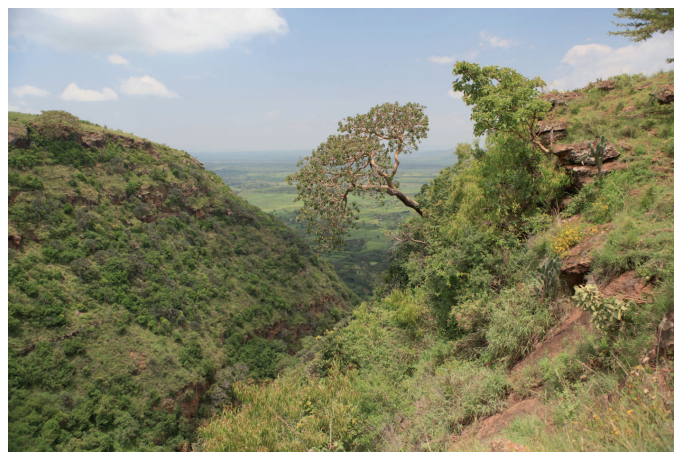

Blick in den Grabenbruch in der Nähe von Asella

den Lake Ziway, einen der großen Seen im Grabenbruch, überthront.

Der Vielvölkerstaat Äthiopien gliedert sich in neun Regionen, wovon sechs durch eine eigene Ethnie und Sprache gekennzeichnet sind. Asella liegt in Oromia, dem Land der Oromo. Die lokale Sprache nennt sich Oromifaa.

Die Region Oromia teilt sich in Zonen auf, wobei Asella die Hauptstadt der Arsi-Zone ist. Gelegen an einer alten Handelsstraße am östli- 


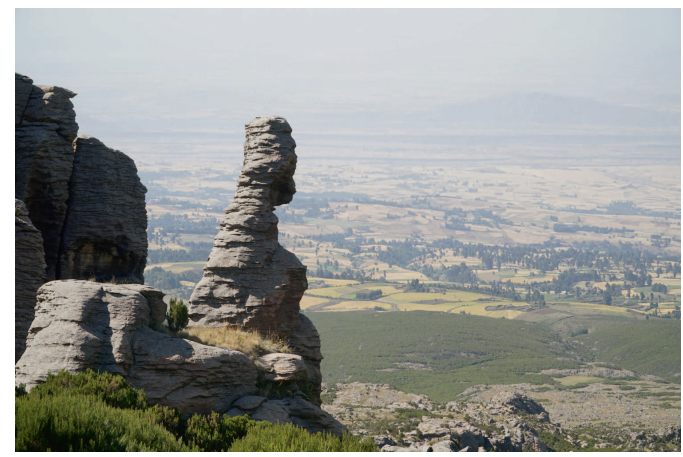

Der "thinking muslim“, eine auffällige Felsformation an den Hängen des Mount Chilalo über Asella

chen Rand des Rift Valleys, entstand hier während der italienischen Besatzungszeit (19351941) eine Provinzhauptstadt. Aus dieser Zeit steht heutzutage in Asella kein Bauwerk mehr, doch in den 60er und 70er Jahren des 20. Jahrhunderts wurden in Asella mit italienischer Unterstützung verschiedene Bauprojekte, wie die Überdachung des Marktgeländes und der Bau des jetzigen Krankenhauses, realisiert.

Die mehr als 110.000 Bewohner Asellas sind sehr religiös. $67 \%$ zählen zu den äthiopischorthodoxen Christen, 23 \% sind Muslime und

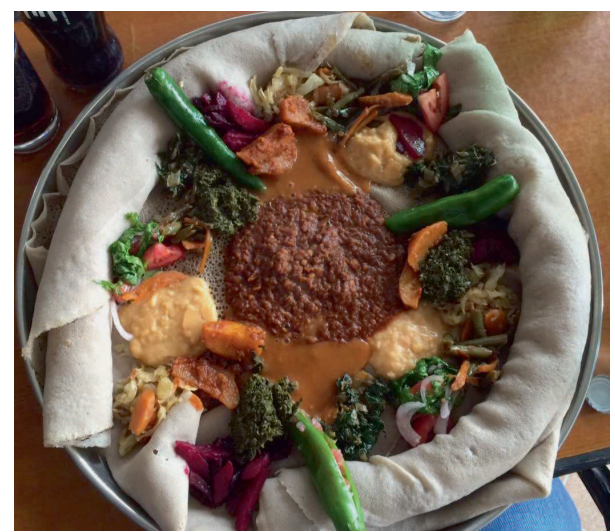

Traditionelle vegetarische Fastenspeise: Beyenet

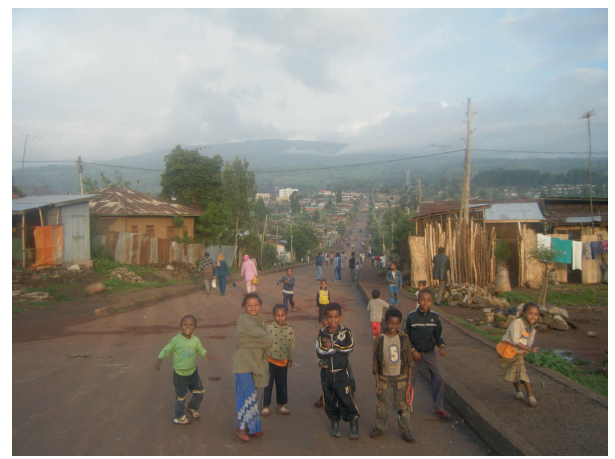

Blick über Asella zum Mt. Chilalo, dem „Hausberg“ der Stadt

mit $9 \%$ stellen Protestanten die wichtigste religiöse Minderheit dar. In Äthiopien kommt es zwischen ethnischen Gruppen immer wieder zu Konflikten. Die Religionsgemeinschaften untereinander pflegen jedoch in der Regel ein sehr tolerantes und respektvolles Miteinander. Obwohl Asella kleinstädtisch oder gar ländlich erscheint, findet man im Straßenbild von modern gekleideten Damen bis zu Kopftuch- oder Burka-Trägerinnen verschiedene Kleidungsstile. In der abendlichen Geräuschkulisse fallen gelegentliche Muezzin-Rufe auf, wie man sie aus arabischen Ländern kennt.

Noch deutlich lauter und länger ertönen jedoch die zu Gebet und Gottesdienst aufrufen-

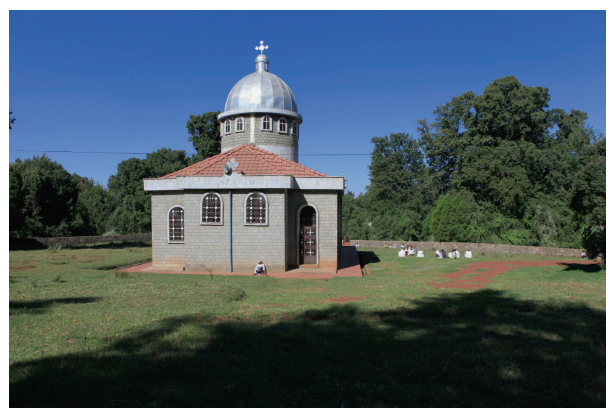

Orthodoxe Kirche in der Nähe von Asella 


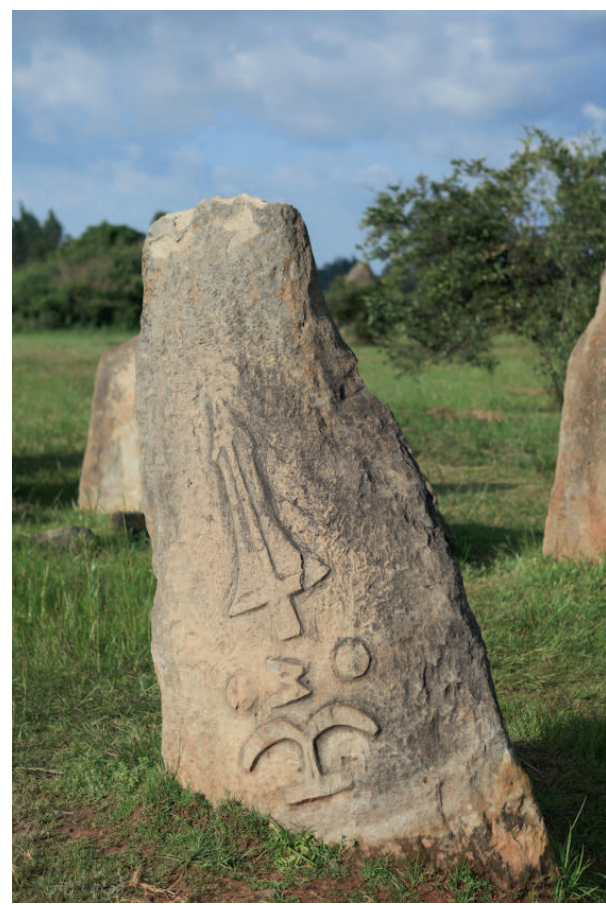

Stele aus vorgeschichtlicher Zeit auf einem der für Oromia typischen Stelenfelder historisch ungeklärten Ursprungs

den Gesänge aus den Lautsprechern der orthodoxen Kirchen - im Rahmen von Feiertagen auch über mehrere Nachtstunden hinweg.

Respekt wird den Religionen auch von staatlicher Seite entgegengebracht. So addieren sich christliche wie muslimische Feiertage im äthiopischen Kalender, und die jeweils nicht zu religiösen Festlichkeiten verpflichtete Bevölkerungsgruppe erfreut sich an entsprechenden arbeitsfreien Tagen.

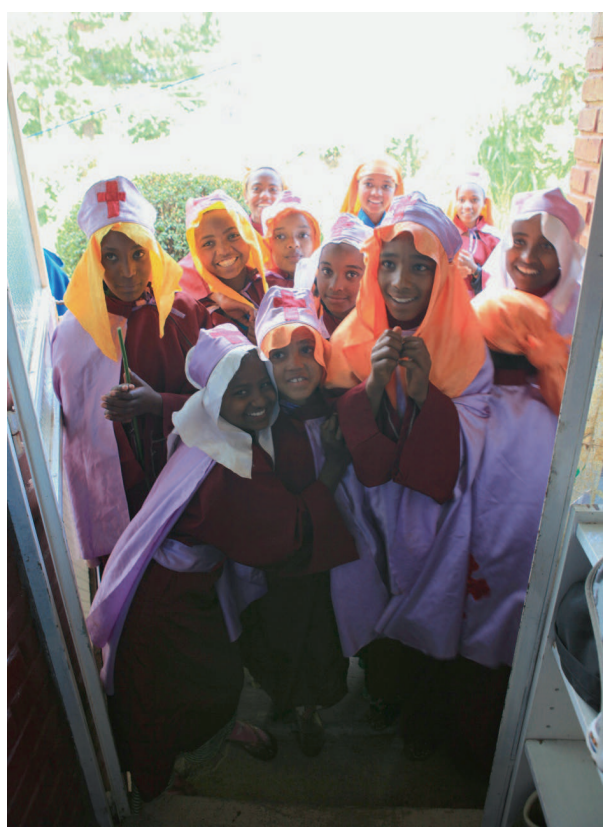

Das orthodoxe Neujahrsfest fällt meist auf unseren 11. September. Kinder ziehen von Haus zu Haus und sammeln Kleingeld und Süßigkeiten

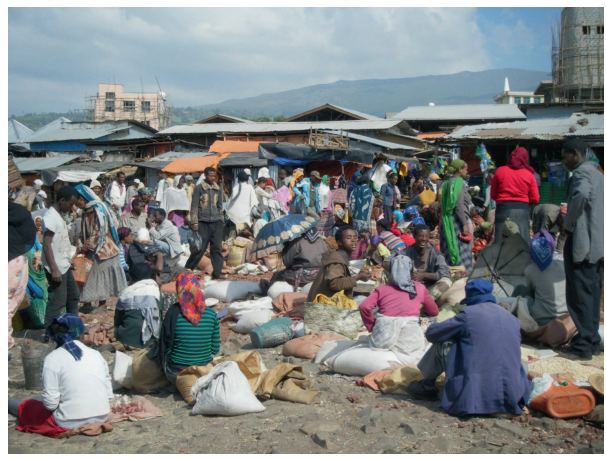

Markt in Asella 


\section{Verkehr und Fortbewegung}

Von Adama kommend, erreicht man Asella nach ca. einstündiger Fahrt und einem stetigen Anstieg über 1.000 Meter. Zunächst noch im Flachland des hier sehr breiten großen Grabenbruchs passiert man riesige Zuckerrohrplantagen, die künstlich bewässert werden. Hier weiden in der Trockenzeit große Dromedar-Herden, die als Zuchttiere anschließend über Djibouti auf die arabische Halbinsel verkauft werden. Der Awash-Fluss mit seinen saftigen Mangobaumhainen wird überquert. Mit stetigem Anstieg weicht das Grün der Kulturlandschaften zunehmend einer semiariden Halbwüste. Schirmakazien, Kakteen und verstreute Lehmhütten prägen das Bild. Nur während der wenige Monate dauernden Regenzeit blüht das Land kurz auf, und ein sattes Grün dominiert das Land.

Der Reisende passiert Kleinstädte und Dörfer. Der Großteil der Felder wird durch Kleinbauern bestellt, die mit ihren Familien in Lehmhütten zwischen den Feldern leben. An der Straße entlang reiten Kinder auf Eseln, bepackt mit gelben Plastikkanistern, in denen sie Wasser von öffentlichen Brunnen nach Hause transportieren. Die Straße von Ada-

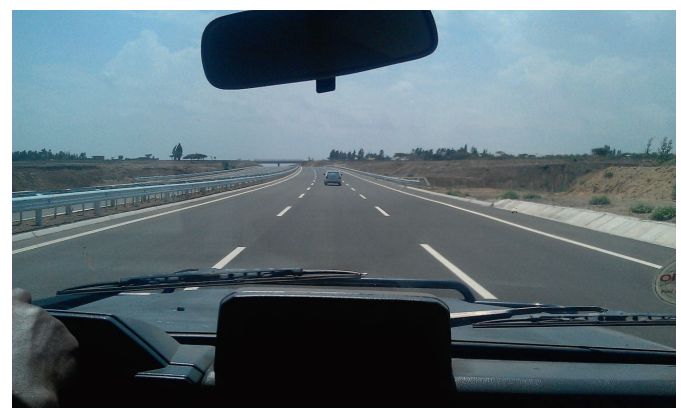

Seit Einweihung der neuen, gebührenpflichtigen Autobahn hat sich die Fahrtzeit von Addis Abeba nach Adama um Stunden verkürzt ma nach Asella und darüber hinaus wurde vor einigen Jahren im Rahmen der chinesischen Entwicklungszusammenarbeit zweispurig ausgebaut und weist im Vergleich zum Landesdurchschnitt relativ wenige Schlaglöcher auf. Vollbeladene Eselskarren oder sich den Berg hinauf schleppende, schwer beladene LKW scheinen auf der Fahrbahn zu stehen und zwingen zu vielen Überholmanövern. Die dort häufig genutzten Transporter der Marke Isuzu transportieren auf ihren offenen Ladeflächen hoch gestapelte Güter, ganze Rinderherden oder auch, eng gedrängt, Menschen. Als stärkste Vehikel der Straße scheinen sie nie für andere bremsen zu müssen. Überholt wird man im PKW häufig von den im Straßenbild zahlreich anzutreffenden, meist überbesetzten Minibussen. Mit diesen bewerkstelligen Kleinunternehmer den Personenverkehr zwischen den Städten Äthiopiens und versuchen durch Überladung, Sparen an Sicherheit im Fahrzeug, beständig überhöhte Geschwindigkeit und riskante Fahrmanöver ihren Profit zu maximieren.

Die Mitarbeiter des Instituts müssen nicht auf den unsicheren öffentlichen Transport zurückgreifen: In den institutseigenen Pkws, einem Nissan Patrol, Baujahr 2000, und einem fabrikneuen Toyota Landcruiser, Baujahr 2017, erfolgt die Beförderung sicher und in gemäßigtem Tempo.

Die Straße von Adama Richtung Süden zieht sich an der östlichen Kante des Rift Valley entlang und durch Asella, an den Bergmassiven des Mt. Chilalo und Mt. Kaka vorbei, in die nahen Bale Mountains im Osten und nach Westen wieder hinab in das Rift Valley zu den Städten Awassa und Shashemene, der Hochburg der Rastafaris in Äthiopien. 


\section{Wohnen in Asella}

Mit dem Kooperationsvertrag sagte die Adama-Universität eine adäquate Unterbringung für die entsandten Kollegen aus Düsseldorf zu. In der Praxis wurde diese Zusage zunächst durch ein Haus realisiert, welches sich im Besitz der Universität befindet und in Ardu liegt. Da seit 2013 meist zwei Düsseldorfer Mitarbeiter vor Ort sind, wurde dann zusätzlich auch das baugleiche Nachbarhaus zur Verfügung gestellt.

Ardu, ein Vorort Asellas, befindet sich ca. vier Kilometer vom Krankenhaus von Asella und dem Institut entfernt, am Hang zum großen Grabenbruch. Nach drei Kilometern auf der

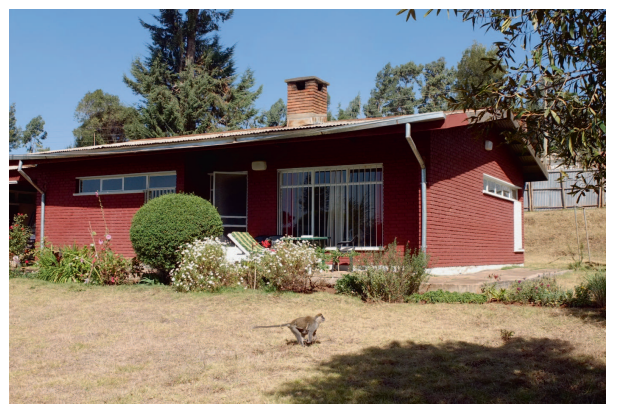

Eines der Wohnhäuser in Ardu

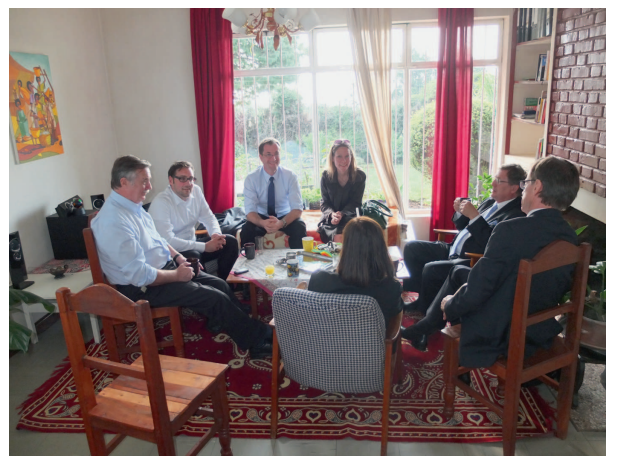

Im Innern des Wohnhauses

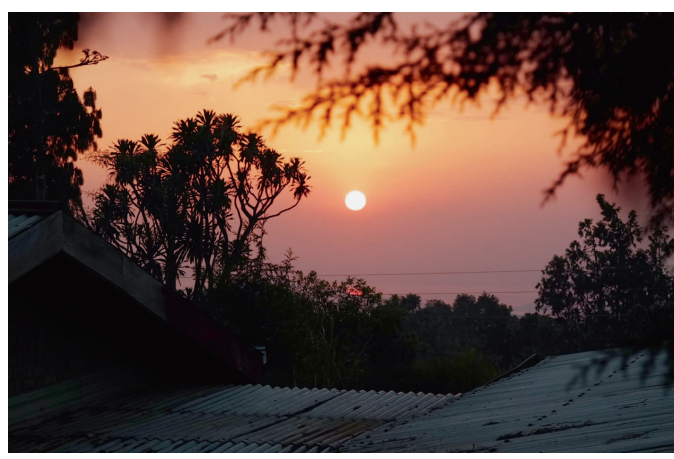

Der Blick über das Wohnhaus in den großen Grabenbruch - in den Abendstunden ein Genuss

Hauptstraße bergauf biegt man rechts ab, passiert einen Eukalyptus-Wald und ein in die Jahre gekommenes „Naherholungsgebiet“ mit idyllischem See und Modell-Biofarm.

Die kürzlich neu asphaltierte Straße führt zum Verwaltungsgebäude der Arsi-Universität und zur hangabwärts gelegenen agrarwissenschaftlichen Fakultät. Nachdem man eine einfache Wohnsiedlung mit Holz- und Lehmhütten durchquert hat, gelangt man rechts in ein eingezäuntes Areal aus zwei Straßen, auf dem Mitarbeiter eines schwedischen Entwicklungshilfeprojektes in den 60er Jahren in skandinavischem Stil verhältnismäßig großzügige Einfamilienhäuser gebaut haben. In den Häusern, welche von der örtlichen Bevölkerung als „Ardu Villas“ bezeichnet werden, wohnen derzeit Professoren und Verwaltungsangestellte der Universität. Ein Clubhaus und eine Sportanlage mit Tennis-, Squash- und Basketball-Platz zeugen von komfortablen Zeiten einer europäischen Gemeinschaft. Tennis und Squash kann man schon lange nicht mehr auf dem Areal spielen. Der geteerte Basketballplatz wurde inzwischen von einem deutschen Nachbarn, der für die GIZ (Gesellschaft für Inter- 
nationale Zusammenarbeit und Entwicklungsorganisation des Bundes) arbeitete, zusammen mit einem amerikanischen Peace Corps Volunteer instand gesetzt und wird von den Kindern und Jugendlichen der Umgebung täglich bespielt. Nachdem das etwas in die Jahre gekommene Clubhaus zeitweise leer stand, dient es aktuell wieder als Treffpunkt für Studenten und Universitätsmitarbeiter; es werden Kaffee, Tee und typische äthiopische Gerichte serviert.

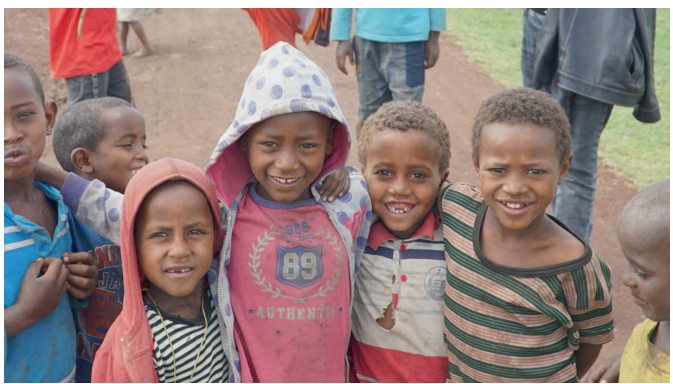

Junge Nachbarn. Trotz alltäglicher Präsenz erregen wir „Weißen“ auf der Straße immer noch viel Aufmerksamkeit

Die aneinandergrenzenden Bungalows aus rotem Backstein, alleinstehend auf schönem Grundstück mit Rasen- und Gartenfläche, sind im äthiopischen Vergleich luxuriös. Küche, Wohnzimmer und zwei Schlafzimmer sind praktisch geschnitten. Ein Kamin im Wohnzimmer ist zumindest dekorativ. Im Hinblick auf die katastrophalen Auswirkungen der Abholzungen in Äthiopien für Brennholz, wird er nur selten in den besonders kalten Nächten während der Regenzeit benutzt.

Die anfangs etwas spärlich möblierten und durch langen Leerstand ein wenig heruntergekommen wirkenden Bungalows wurden nach und nach instand gesetzt.
In der Zwischenzeit richteten sich die Düsseldorfer Ärzte in den Häusern zunehmend auf einen längeren Verbleib ein. Eine kleine Bibliothek für die langen Abende der Regenzeit wächst mit jedem Besucher. In einem angelegten Garten sind heimische Gemüsesorten „affensicher" unter einem Drahtkäfig gepflanzt. Ein Grill für die gemeinsamen Sommerabende wurde aus gefundenen Ziegelsteinen aus der schwedischen Zeit und einem ausgefrästen Stück Blech einer alten Landmaschine gemauert. Regale und Gartenmöbel wurden teilweise selbst gezimmert oder von lokalen Schreinern aus Bambusholz angefertigt. Fernseher aus Deutschland und ein mittlerweile eingerichteter Satellitenempfang erlauben es, dass die Mitarbeiter vor Ort die Geschehnisse in der Heimat nicht völlig aus den Augen verlieren. Jeder neue Mitarbeiter aus Deutschland trägt etwas zur Verbesserung der Wohnlichkeit vor Ort bei.

In dem nicht weniger schönen Blumengarten wurden rasch eine Schaukel und eine kleine Feuerstelle für die Kinder der Familien der ersten Düsseldorfer Bewohner des Hauses angelegt.

Ein Durchgang durch die hohe Hecke verbindet beide Grundstücke, so dass auch nach Feierabend ein reger kollegialer und freundschaftlicher Austausch möglich ist.

Aus Sicherheitsgründen wird ein Nachtwächter beschäftigt. Gesagne, oder richtiger „Ato Gesagne“ („Ato" = amharisch: Ehrwürdiger Herr) ist ca. 60 Jahre alt und diente einst als Soldat in den Eritrea-Kriegen. Er ist herzensgut, hilfsbereit und immer sehr wachsam. Wenn man vor dem Zubettgehen noch einmal hinter dem Haus den wunderbar klaren Blick auf die leuchtende Milchstraße genießt, muss 


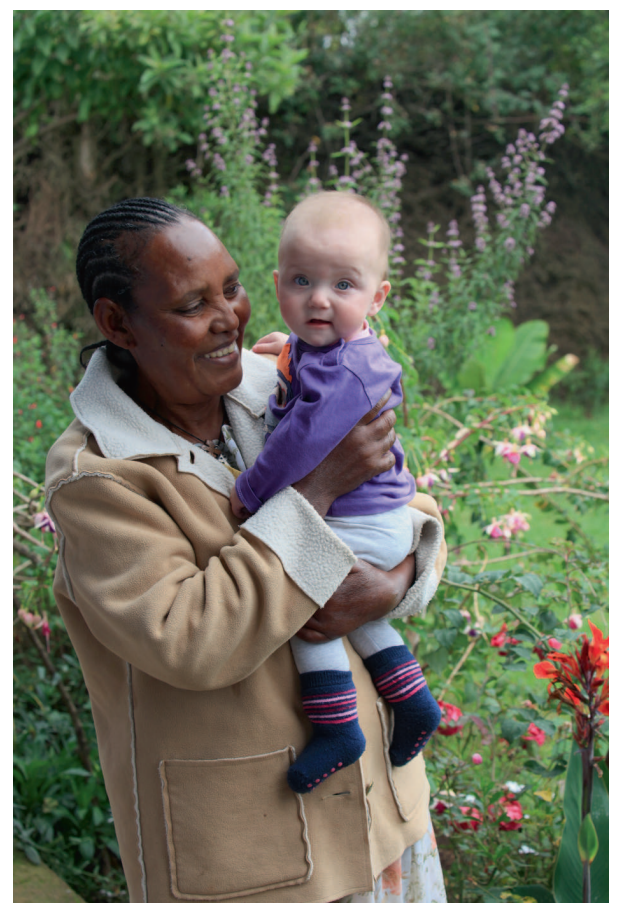

Interkultureller Zusammenhalt: Die Haushälterin Isgaharia mit Clara, der Tochter von Dr. Orth

man sich hüten, unbedacht um die Hausecke zu laufen: Es kann vorkommen, dass man bei dieser Gelegenheit Ato Gesagne begegnet, der durch das leise Geräusch alarmiert wurde - mit hellwachen Augen und einer pinkfarbenen $\mathrm{Pu}-$ delmütze auf dem Kopf, eine lange Machete in der Hand. Sein Sohn Tesfa, ein eher schweigsamer und zurückhaltender Charakter, wurde als Nachtwächter für das zweite Haus eingestellt. Er liebt es, die Stille der kurzen Abende und der frühen Morgenstunden zu genießen.

Tagsüber wachen Kidist und Etaferahu über die Häuser. Sie waschen die Wäsche, spülen Geschirr, putzen und räumen auf. Da es kei- ne gemeinsame Sprache gibt, funktioniert die Kommunikation primär mit Zeichensprache, und die Zusammenarbeit basiert zumindest zu Beginn auf der Akzeptanz, dass sie so Ordnung schaffen, wie sie es für richtig halten. Dazu gehörte z. B. auch, dass Kochgeschirr dekorativ im Wohnzimmerschrank arrangiert wurde.

2013 wurde ein Teil des hinteren Gartens das Zuhause von fünf Hühnern. Der Luxus eines Holzhauses mit Auslauf wurde deutlich erweitert durch die Möglichkeit, sich tagsüber im hinteren Teil des Gartens frei zu bewegen.

So idyllisch das Wohnhaus gelegen ist und so gerne die ehemaligen Bewohner an die Sonnenuntergänge über dem Rift Valley und das Blütenmeer des Gartens zurückdenken, die Wohngegend hat auch Nachteile: Aufgrund der häufigen Stromausfälle vergehen einige Abende bei Kerzenschein, und für den Fall der selteneren, aber meist längeren Wasserausfälle wurden Wassertanks im Garten installiert, welche zumindest zeitweise helfen, den Mangel zu überbrücken. Auch der mobile Internetzugang funktioniert nur lückenhaft.

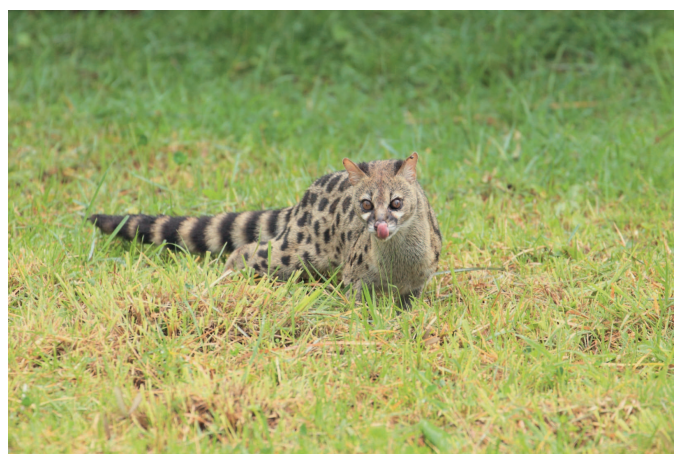

Ginsterkatze im Garten eines der Wohnhäuser 


\section{Zu einer anderen Zeit ...}

Der äthiopische Kalender ist eine Variante des koptischen Kalenders. Er hat zwölf Monate zu je 30 Tagen und einen 13. Monat mit fünf, bzw. in Schaltjahren sechs Tagen. Ethiopian Airlines wirbt mit "13 months of sunshine".

Unserem gregorianischen Kalender hinkt der äthiopische Kalender um über sieben Jahre hinterher. Der 01. Januar 2015 in Düsseldorf war der 23. April 2007 in Asella. Daher muss bei jeder internationalen Kommunikation und Terminabsprache verständlich gemacht werden, ob die Zeitrechnung nach dem internationalen oder äthiopischen Kalender erfolgt. Auch die Tageszeit wird anders angegeben. Aufgrund der Nähe zum Äquator sind Tag und Nacht nahezu konstant 12 Stunden lang, beginnend jeweils um 6:00 Uhr unserer Zeit. Gut verständlich, dass man die Stunden ab Sonnenaufgang oder -untergang zählt. 2:00 Uhr in „local time“ ist also 8:00 Uhr in „international time“, was ebenfalls bei jeder Kommunikation verdeutlicht werden sollte, um Missverständnisse zu vermeiden. Dem, aller mühevoller Absprachen zum Trotz, unterschiedlichen Verständnis von Zeit ist allerdings zuzuschreiben, dass man bei einer Verabredung um 2:00 Uhr "local time" doch am Ende meist erst einmal alleine ist. Der äthiopische Partner kommt fröhlichfreundlich ... zu einer anderen Zeit.

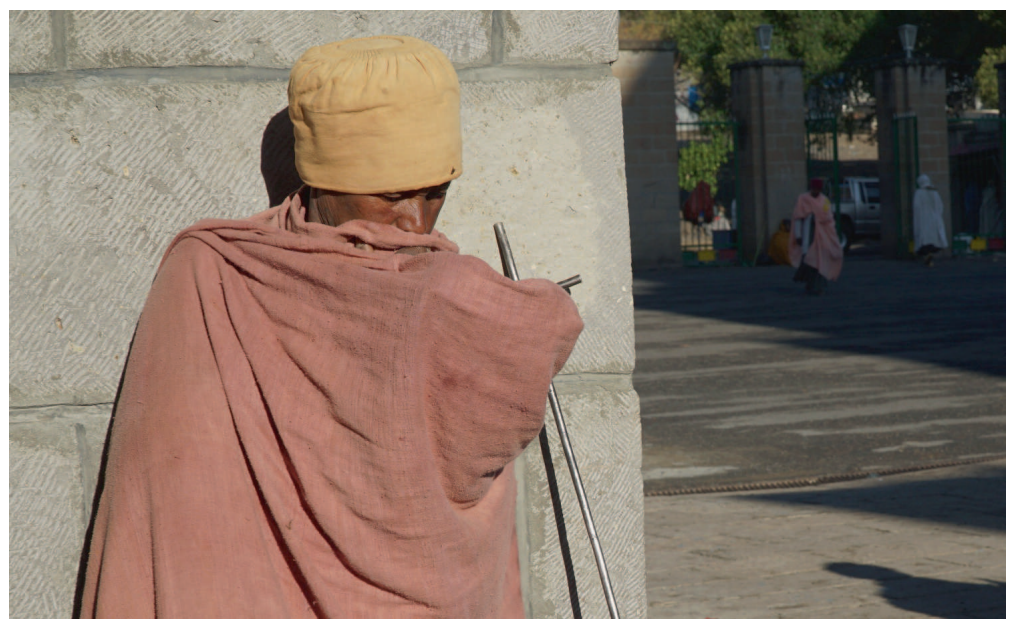

Traditionell gekleideter orthodoxer Mönch in Addis Abeba 


\section{Die fünfte Jahreszeit: Ernte}

Das Tropeninstitut liegt inmitten der sehr fruchtbaren Arsi-Zone, der Getreidekammer Äthiopiens. Neben Weizen werden hier an den Hängen des Mt. Chilalo vor allem Teff (Zwerghirse), Hauptbestandteil der aus der äthiopischen Küche nicht wegzudenkenden Sauerteigfladen „Injeera“, und Gerste für die Produktion des lokalen Biers „T'ela“, aber auch Hirse, Mais, Bohnen, Hafer und verschiedene Linsensorten angebaut. Die Ackerbautradition reicht sehr weit zurück. Viele der in Europa und den USA kultivierten Weizensorten haben in Äthiopien ihren Ursprung. In Äthiopien erfolgt der Getreideanbau nur zu einem geringen Teil auf dem Boden riesiger Staatsfarmen und in den seltensten Fällen mechanisiert. Landesweit weitaus häufiger findet man Kleinbauern, die ihre ca. 1 Hektar großen Felder mit Hilfe des Einsatzes von Nutztieren bestellen. Die verschiedenen Getreidepflanzen keimen nach der Aussaat während der Regenzeit (Juni/Juli) aus. Im Anschluss an den Regen folgt die über Monate andauernde Trockenzeit, während der das Korn ausreift. Die Ernte ist nur mit Hilfe zahlreicher Erntehelfer zu bewerkstelligen. Die Ähren werden dabei meist mit Sicheln gemäht und gesammelt. Auf Dreschplätzen verrichten Ochsen ihre Arbeit als lebende „Dreschflegel“ - in kleinen Gruppen werden die Tiere immer wieder über die verstreuten Ähren getrieben, bis sich alle Körner vom Stroh und der Spreu gelöst haben. Die weiteren Schritte sind meist Frauenarbeit: das Aussieben der Körner und das Aufschichten des Strohs zu großen Garben als Viehfutter für die Trockenzeit. Abgepackt in Säcke und hoch aufgetürmt auf Eselskarren, Pferdekutschen und LKWs verschwindet das Korn schließlich in den Hütten der Bauern und den großen Lagerhäusern der Händler. Die Erntezeit ist dabei für die Landbevölkerung von so großer Bedeutung, dass sogar die Fallzahlen für verschiedene Erkrankungen in Krankenhäusern und kleineren Gesundheitsstationen rückläufig sind. Die Menschen nehmen sich während dieser wichtigen Wochen keine Zeit für die eigene Gesundheit. Das Land mit seinen Bewohnern passt sich gleichsam dem stets wiederkehrenden Rhythmus aus Trocken- und Regenzeit, Aussaat und Ernte an. 


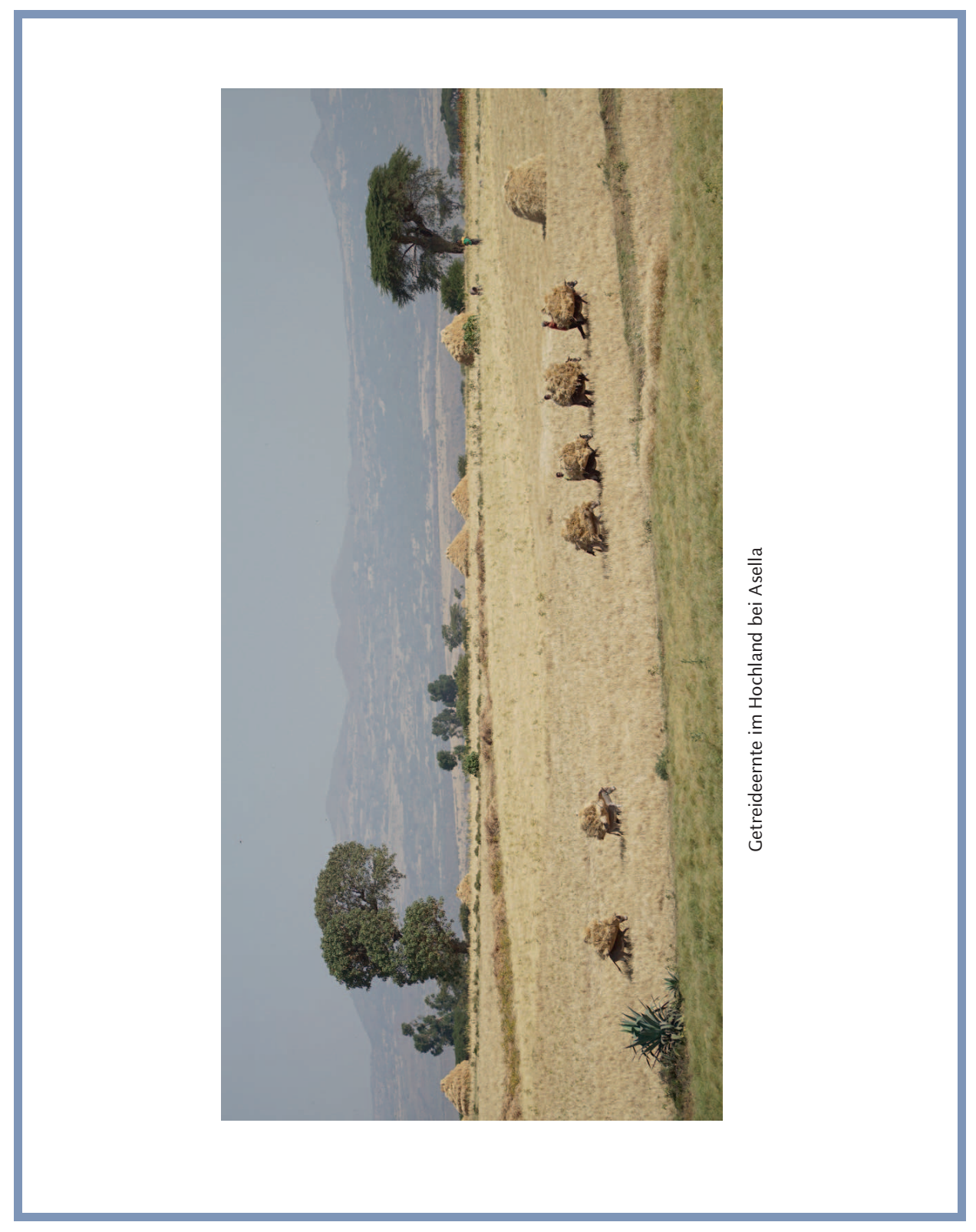




\section{Dürre}

Nachdem sich Ende September die letzten vom indischen Ozean heranziehenden Regenschauer verzogen haben, beginnt die lange Trockenperiode. Die zu Beginn der Trockenzeit noch grünen Getreidefelder werden als gelbe und trockene Pflanzen in den Wintermonaten abgeerntet. Das voller Leben steckende Land wandelt sich. Bäche und Flüsse werden zu steinigen Gräben. Ganze Seen voller farbenfroher Wasservögel versanden und verwandeln sich im Laufe der trockenen Wochen und Monate in öde Wüsten. Aus den trockenen Ebenen im Osten des Landes treiben nomadisch lebende Afar und Somalis ihre Kamelherden auf der Suche nach Wasser und Nahrung in das höher gelegene Bergland in Oromia. Während der Regenzeit prächtig und kräftig anzuschauende, aber jetzt bis auf die Knochen abgemagerte Rinder knabbern kraftlos an auf den Feldern vergessenem Stroh. Allgegenwärtige Tüpfelhyänen verirren sich auf der Suche nach Nahrung immer häufiger mitten in Dörfer und kleinere Städte. Zum Höhepunkt der Trockenzeit ziehen Staubtornados durch die Tiefebenen des großen Grabenbruchs, und heiße Winde treiben ihre trockene Last durch jeden Spalt und jede Lücke in Häuser und Autos; fast alles wird durch eine rötliche Staubschicht bedeckt. Buschfeuer toben durch die Heidelandschaft in den Höhenlagen der über 4.000 m hohen Berge. Bedingt durch die Dürre häufen sich in dieser Zeit die Ausfälle der städtischen Wasserversorgung, so dass das Krankenhaus in Asella zeitweise seine OP-Kapazität einschränken muss, da nicht mehr ausreichend Wasser zur Vorbereitung der Instrumente zur Verfügung steht. Derzeit wird daher ein großes Wasserreservoir für die Versorgung des Krankenhauses errichtet.

Im Frühjahr und mit einsetzenden Westwinden werden endlich tropische Regenwolken aus dem Kongobecken in Richtung Äthiopien getrieben. Von Süden her kommt Regen auf. Aus großen Kumuluswolken ergießen sich Platzregen über die ausgetrocknete Erde. Schwere Gewitter ziehen durch das Rift Valley. Innerhalb weniger Tage erwacht die Natur zu neuem Leben. Die Bauern strömen auf die Felder, um mit dem Pflug den nun erweichenden Boden zu bearbeiten. Rinder, Pferde, Esel, Schafe und Ziegen fressen sich am frischen Grün satt. Die jährliche Trockenheit ist überwunden. 


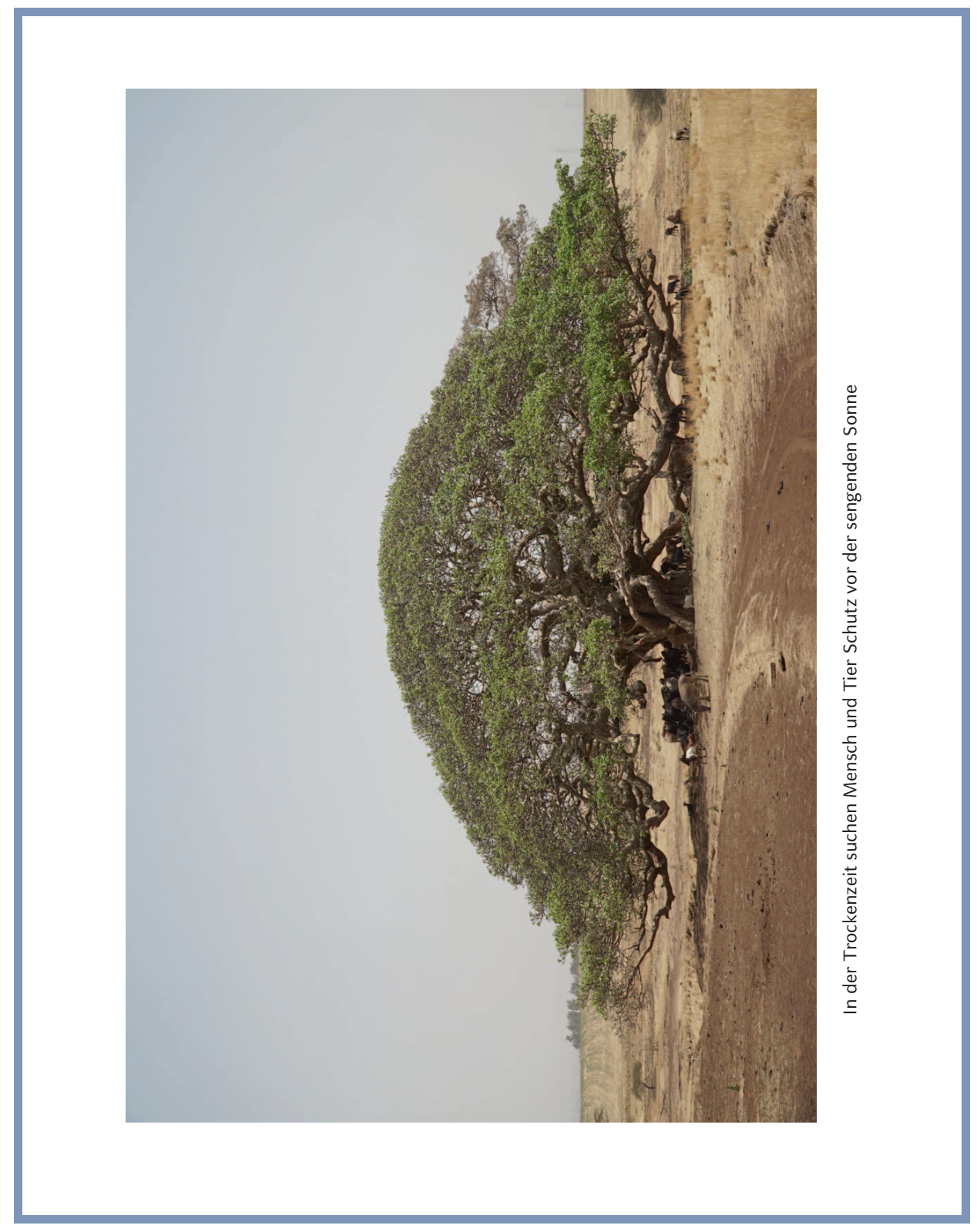




\section{Unter Deutschen}

Nicht gerade eine Metropole, dieses Asella. Die internationale Community im Städtchen ist überschaubar. Zwei bis drei Koreaner arbeiten in einem Projekt von KOICA, der südkoreanischen Entwicklungshilfeorganisation, vor den Toren der Stadt. Einige Inder und Kubaner sind als Lehrer an einer der verschiedenen Berufsschulen tätig. Einige US-Amerikaner leisten im Rahmen des Peace Corps Hilfe im ländlichen Äthiopien. Ein Franzose widmet sich der Rettung des äthiopischen Wolfes in den Bergen rund um Asella und chinesische Bauunternehmen errichten neben einzelnen Straßen auch das neue Stadion für die Athleten der Sportakademie.

Und dann leben in Asella noch wir, einige Deutsche. Bis in die erste Hälfte des Jahres 2015 gab es da noch einen Mitarbeiter der GIZ aus Dortmund, ein bekennender Borussia-Fan. Sieben Jahre lang hat der gelernte Landmaschinenmechaniker und studierte Sozialarbeiter für verschiedene Entwicklungshilfeorganisationen in Äthiopien gearbeitet. Zuletzt war er für die Organisation eines landwirtschaftlichen Trainingszentrums der GIZ auf dem Gelände einer nahegelegenen Staatsfarm verantwortlich, welches es sich zum Ziel gesetzt hat, die Mechanisierung der Landwirtschaft voranzutreiben. Professor Dietrich Birnbaum, Emeritus aus der Herzchirurgie, der leider 2017 verstorben ist, und Professor Frank Riedel, Emeritus aus der Kinderheilkunde, haben bis 2016 jeweils für mehrere Monate im Jahr das Krankenhaus in Asella und die Medizinische Fakultät durch Expertenwissen, Lehre und praktische Unterstützung in der Behandlung der Patienten unterstützt. Organisiert wurde dieser Austausch durch den DAAD. Zu Besuch kam auch Professor Dr. Eberhardt Becker, emeritierter Professor für Algebra und Rektor der Technischen Universität Dortmund. Am Ende bleiben noch die beiden Ärzte des HITM zu erwähnen, die mit Familie oder Partnern in Asella leben. So entsteht schnell ein vertrautes Miteinander, man kennt sich, hilft einander und tauscht nicht nur Informationen aus aller Welt, sondern auch Spezialitäten aus der jeweiligen Heimat untereinander aus. Besonders hoch im Kurs stehen würziger Schinkenspeck, Müsli und natürlich Schokolade. Zusammen mit den Kindern eines deutschen Kollegen wurde im November 2014 der wohl erste Martinszug in Asella abgehalten, und zu Ostern werden kleine Geschenke und Ostereier versteckt. So lebt man hier, im Ausland, mitten in Äthiopien und am Ende irgendwie doch - unter Deutschen. 


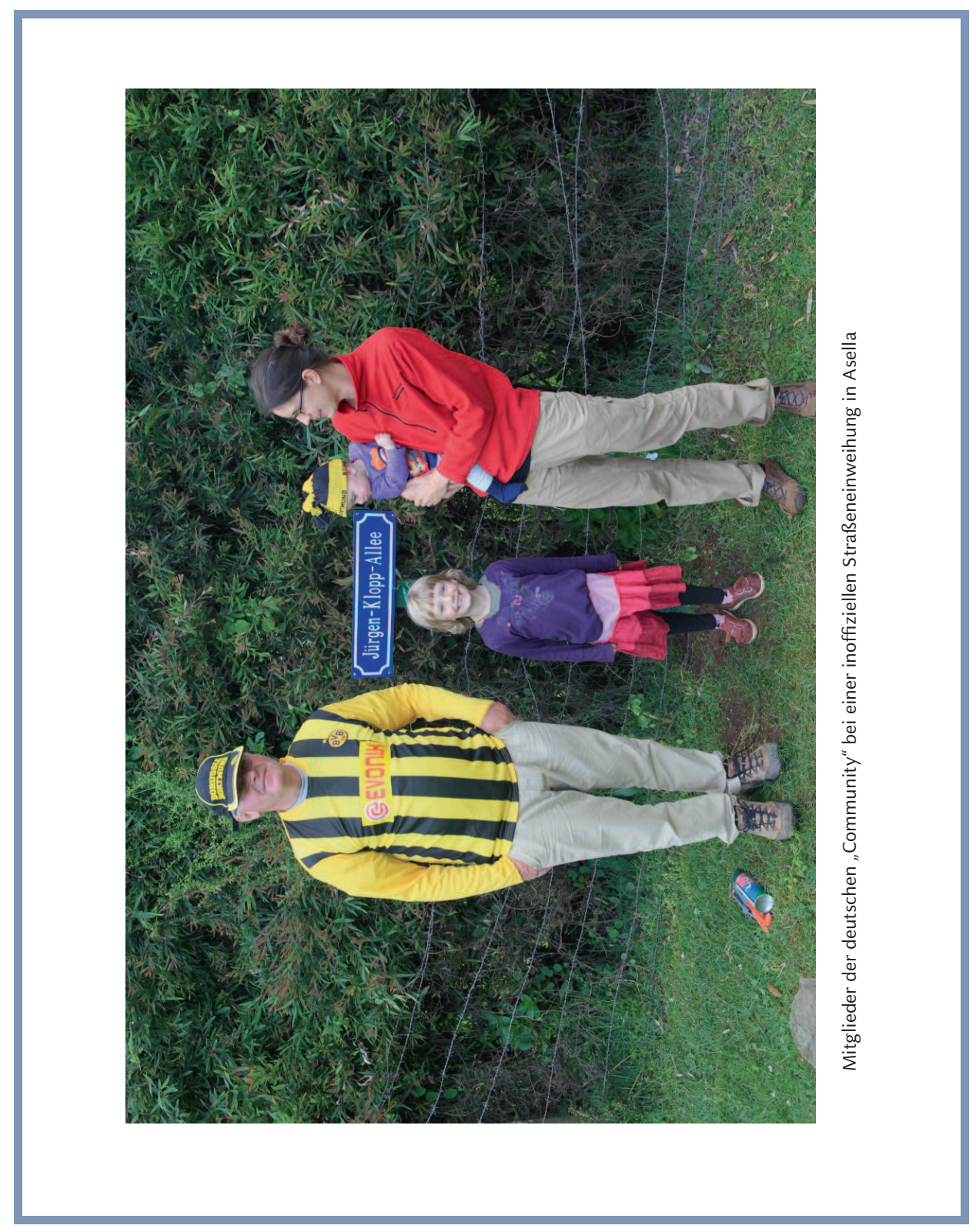




\section{Flöhe}

Die IUCN (International Union for the Conservation of Nature and Natural Resources) hat ihn noch nicht auf die rote Liste gesetzt, obwohl er weltweit zunehmend seltener wird. In Äthiopien hingegen erfreut sich Pulex irritans, der Menschenfloh, seines Daseins. Die lückenhafte Versorgung mit Leitungswasser und das enge Zusammenleben zwischen Mensch und Tier bieten ihm optimale Lebensbedingungen.

Die Einheimischen sind an die Flöhe gewöhnt und reagieren - wenn überhaupt - nur mit leichtem Juckreiz auf Flohstiche. Es sind insbesondere die „Faranjis“, also weiße Ausländer wie wir, welche die Flöhe als Plage empfinden. Wenn zunächst leichter Juckreiz einsetzt, ist der Floh längst wieder verschwunden. Erst Stunden später ist das gesamte Ausmaß sichtbar: Mehrere gerötete Quaddeln nebeneinander fangen zunehmend an zu jucken. Übliche Insektenstich-Salben helfen einigermaßen, mit Insektenschutzmitteln lässt sich das Problem leidlich gut eindämmen.

Die Flöhe sind nur etwa $2 \mathrm{~mm}$ groß, sie bewegen sich lautlos und können bis zu $50 \mathrm{~cm}$ weit springen. Echte Akrobaten also. Dementsprechend gelingt es so gut wie nie, einen Floh dingfest zu machen. Nach bestimmten anatomischen Kriterien ist der Menschenfloh taxonomisch eindeutig vom Rattenfloh, dem Hauptüberträger der Pest, zu unterscheiden. Zum Glück ist Pulex irritans nur in sehr geringem Umfang in der Lage, Krankheiten zu verbreiten.

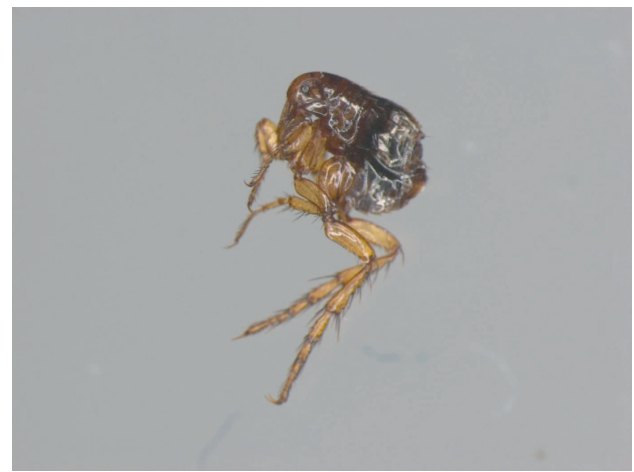

Äthiopischer Menschenfloh 